\title{
Besonderheiten bei der Ozonolyse substituierter Cyclopentene
}

Kurt Schank, Susanne Pistorius, Matthias Weiter und Frank Werner

Saarbrücken, Fachrichtung 11.2 Organische Chemie der Universität des Saarlandes

\section{Eingegangen am 17. Januar 1995}

Details on Ozonolyses of Substituted Cyclopentenes

In ihrer Studie über "Intramolekulare Konkurrenzreaktionen bei der Ozonolyse substituierter Cyclopentene" gingen Criegee und Mitarbeiter [1] aufgrund früherer Erfahrungen [2] davon aus, daß Cyclopentene $1\left(\mathrm{R}^{1}, \mathrm{R}^{2}=\mathrm{H}\right)$ mit $2 \mathrm{H}$-Atomen an der Doppelbindung unter den üblichen Alkenozonolysebedingungen in Lösung nicht mehr zur Bildung monomerer Ozonide neigen [3]. Demgegenüber wurden aber aus offenkettigen Alkenen immer dann hohe Ozonidausbeuten erhalten, wenn beide C-Atome der Doppelbindung (wie in Cyclopenten 1a selbst) noch mindestens $1 \mathrm{H}$-Atom gebunden hatten [4]. Dagegen lieferte in der offenkettigen Reihe Tetramethylethylen (2,3-Dimethyl-but-2-en) kein Ozonid mehr [5]. Völlig überraschend jedoch lieferte das dazu analoge 1,2-Dimethylcyclopenten (1i) das monomere Ozonid $\mathbf{2 i}$ in 80-proz. Ausbeute [6] (Schema 1).

Schema 1 Bislang vorausgesetzte Forderungen für die Bildung isolierbarer monomerer Alkenozonide bei der Ozonolyse in Lösung

a)

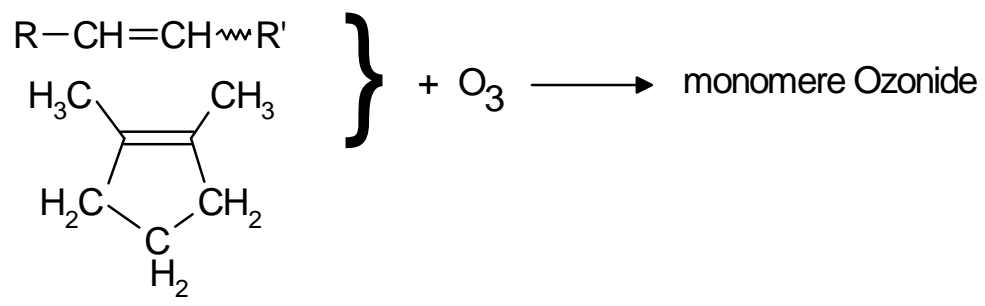

1i

b)

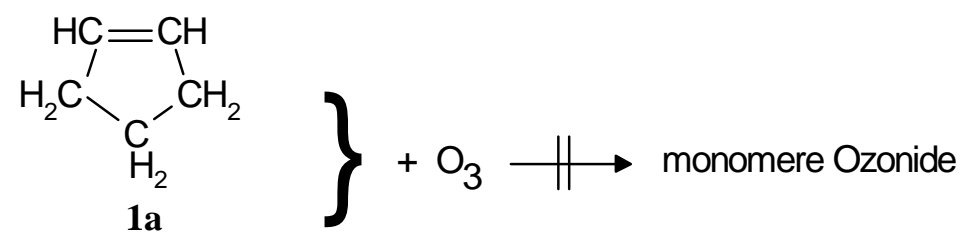<smiles>CC(C)=C(C)C</smiles> 
Unter den Gesichtspunkten des Criegee-Mechanismus [7] sind diese unterschiedlichen Ergebnisse kaum erklärbar, denn danach sollten Aldehydcarbonylgruppen und Aldehydcarbonyloxide aufgrund höherer Reaktivität immer leichter erfolgende Cycloadditionen zu Ozoniden zeigen als Ketoncarbonylgruppen und Ketoncarbonyloxide. Gleichermaßen überraschend lieferte das an der Doppelbindung monosubstituierte 1-Phenylcyclo-pent-1-en (1b) sogar bei Raumtemp. in $\mathrm{CCl}_{4}$ ein stabiles monomeres Ozonid $\mathbf{2 b}$ in fast quantitativer Ausbeute [8]. Der ebenfalls an der Doppelbindung monosubstituierte Cyclopent-1-en- 1-carbonsäuremethylester 1c [9] lieferte das entsprechende Ozonid 2c in Frigen 11 bei $-70^{\circ} \mathrm{C}$ zwar ebenfalls gemäß titrimetrischer Peroxidbestimmung und spektroskopischer Analyse [10] in quantitativer Ausbeute, eine Isolierung bei Raumtemperatur wie bei $\mathbf{2 b}$ war jedoch infolge Instabilität nicht mehr möglich.

Wir fanden nun bei vergeblichen Versuchen zur Darstellung von 2,7-Dioxadekalin-1,8-dion (quasi eine cis-Meldrumsäure [11]) ein unerwartetes Ergebnis. Aus Cyclopent-3-en-1-ol [12] wurden dessen Derivate 1d-h hergestellt und bei $-78^{\circ} \mathrm{C}$ in Frigen 11 oder Methylenchlorid mit einem Ozon/Sauerstoffgemisch umgesetzt (1:1-Stöchiometrie) (Schema 2).

Schema 2 Ozonolyse 4-substituierter Cyclopentene 1d-h und Vergleich mit Literaturbeispielen 1a-c, $\mathbf{i}$

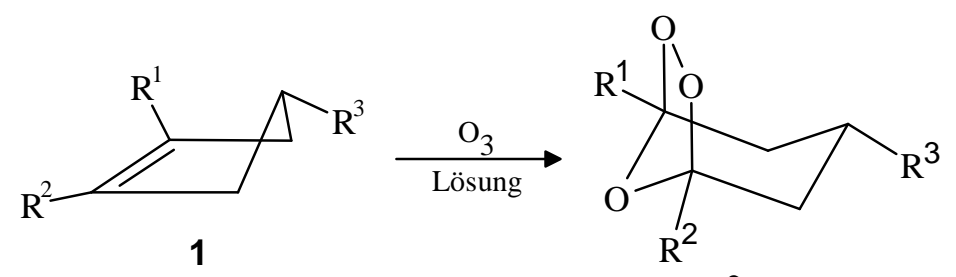

\begin{tabular}{c|ccc}
$\mathbf{1 , 2}$ & $\mathrm{R}^{1}$ & $\mathrm{R}^{2}$ & $\mathrm{R}^{3}$ \\
\hline $\mathbf{a}$ & $\mathrm{H}$ & $\mathrm{H}$ & $\mathrm{H}$ \\
$\mathbf{b}$ & $\mathrm{H}$ & $\mathrm{C}_{6} \mathrm{H}_{5}$ & $\mathrm{H}$ \\
c & $\mathrm{H}$ & $\mathrm{CO}_{2} \mathrm{CH}_{3}$ & $\mathrm{H}$ \\
\hline d & $\mathrm{H}$ & $\mathrm{H}$ & $\mathrm{OCOC}_{6} \mathrm{H}_{5}$ \\
$\mathbf{e}$ & $\mathrm{H}$ & $\mathrm{H}$ & $\mathrm{OCOC}_{6} \mathrm{H}_{4}-4-\mathrm{NO}_{2}$ \\
$\mathbf{f}$ & $\mathrm{H}$ & $\mathrm{H}$ & $\mathrm{OSO}_{2} \mathrm{C}_{6} \mathrm{H}_{4}-4-\mathrm{Cl}$ \\
g & $\mathrm{H}$ & $\mathrm{H}$ & $\mathrm{CH}\left(\mathrm{CO}_{2} \mathrm{CH}_{3}\right)_{2}$ \\
$\mathbf{h}$ & $\mathrm{H}$ & $\mathrm{H}$ & $\mathrm{CH}\left(\mathrm{CO}_{2} \mathrm{C}_{2} \mathrm{H}_{5}\right)_{2}$ \\
\hline $\mathbf{i}$ & $\mathrm{CH}_{3}$ & $\mathrm{CH}$ & $\mathrm{H}$
\end{tabular}


Ebenso wie bei $2 \mathrm{c}$ wies die ${ }^{1} \mathrm{H}-\mathrm{NMR}$-spektroskopische Tieftemperaturuntersuchung auch für 1d und 1e die Bildung der Ozonide 2d und 2e aus, beim Versuch der Isolierung gingen diese jedoch schon bei $0^{\circ} \mathrm{C}$ in zähe polymere Peroxide über, die bei Versuchen der chromatograpischen Trennung (stationäre Phase neutrales Aluminiumoxid, mobile Phase Methylenchlorid) auf der Säule Verpuffung zeigten. Demgegenüber konnte das Ozonid $2 \mathbf{f}$ des 4-Chlorbenzolsulfonsäureesters 1f zumindest kristallin erhalten werden, wenngleich es sich $>40^{\circ} \mathrm{C}$ zersetzte. Die aus den Cyclopent-3-en-1-yl-malonestern 1g, $\mathbf{h}$ in quantitativer Ausbeute erhaltenen Ozonide 2g, h folgten auch nicht dem allgemeinen Trend (vgl. Schema 1), daß Cyclopentene ohne Substituenten an der Doppelbindung unter den Bedingungen der Ozonolyse in Lösung bei tiefer Temperatur (hier bei $-78^{\circ} \mathrm{C}$ in Frigen) nicht zur Bildung isolierbarer Ozonide neigen. Die Ozonide 2g, h zeigten eine ungewöhnliche Stabilität: Beim qualitativen Peroxidtest, dem Einbringen einer kleinen Substanzprobe auf einem Metallspatel in eine Bunsenbrennerflamme, erfolgte nicht die sonst übliche Verpuffung, sondern die Ozonide brannten nach dem Schmelzvorgang mit ruhiger Flamme ab. Allgemein werden zwei Syntheseprinzipien für Ozonide beschrieben:

1) [3+2] Cycloaddition eines C,O,O-Fragments (Carbonyloxid) an ein C,O-Fragment (Carbonylgruppe) (Criegee-Schema);

2) $[3+2]$ Cycloaddition eines $\mathrm{C}, \mathrm{O}, \mathrm{C}$-Fragments an ein O,O-Fragment $\left(\mathrm{O}_{2}\left({ }^{1} \Delta_{\mathrm{g}}\right)\right)$ (allgemeine Abfang- und Nachweisreaktion für Singulettdisauerstoff mit Hilfe von Furanen, insbesondere mit Diphenylisobenzofuran (DPBF) [13]).

Cyclopentenozonolysen scheinen intermediär dem 1. Prinzip nicht zu folgen, worauf auch der Befund von Bailey [14] hinweist, daß die Ozonolyse von 1,2-Diphenylinden bei $15^{\circ} \mathrm{C}$ in Cyclohexan nur $64 \%$ Ozonid liefert, während das gleiche Ozonid bei $15^{\circ} \mathrm{C}$ in Eisessig, einem bekannten Abfangreagenz für Carbonyloxide, sogar mit 80\% Ausbeute entsteht. Die dort zugunsten von Prinzip 1 angeführten "besonders günstigen räumlichen Verhältnisse für den intramolekularen Ringschluß zum Ozonid" finden sich in erhöhtem Maß auch bei Norbonen, einem 3,5-verbrückten Cyclopenten, trotzdem bildet dieses unter den Bedingungen der Ozonolyse in Lösung [15] kein isolierbares [16] Ozonid mehr. Dagegen liefert Hexa-trifluormethyl-benzvalen als ebenfalls 3,5-verbrücktes Cyclopenten trotz höherer Ringspannung ein bei Raumtemp. isolierbares Ozonid [17]. Die von Criegee im Epilog seines letzten Beitrags 1975 angesprochenen "vielen offenen Fragen" [7a] konnten bis heute nicht zufriedenstellend beantwortet werden, dazu bedarf es noch weiterer Untersuchungen und deren 
ergebnisorientierter Interpretationen [18], wobei der Substitution in Cyclopentenen bei deren Ozonolyse offenbar eine wichtige Bedeutung zukommt.

Wir danken den Kollegen M. Regitz und G. Maas vom Fachbereich Chemie der Universität Kaiserslautern für die freundliche Unterstützung bei der Anfertigung von Elementaranalysen sowie Herrn Dr. R. Graf für seine engagierten Bemühungen bei Aufnahme und Interpretation von Ozonid-DCI- Massenspektren.

\section{Beschreibung der Versuche}

Schmelzpunkte wurden mittels einer geeichten Koflerbank und im Kupferblock bestimmt. Reaktionskontrollen wurden mittels DC-Fertigfolien Alugram ${ }^{\circledR}$ SIL G/UV ${ }_{254}$ und Polygram ${ }^{\circledR}$ Alox N/UV 254 von Macherey und Nagel durchgeführt, Mikroanalysen mittels eines Carlo-Erba-Geräts (Universität Kaiserslautern) oder nach Walisch [19], IR-Spektren wurden mittels eines Beckman IR 33, ${ }^{1} \mathrm{H}-\mathrm{NMR}-$ Spektren mit den Geräten Varian EM 360, Bruker WH-90 und AM-400, ${ }^{13}$ C-NMR-Spektren am Bruker AM-400 (bei 100 MHz und Breitbandentkopplung) sowie EI-MS-Spektren am Finnigan MAT-311 (70 eV) und DCI-MS-Spektren am Finnigan MAT-90 (120 eV, Isobutan) gemessen. Ozon-Sauerstoffgemische lieferten die Generatoren 503 von Fischer bzw. 301.7 von Sander, Ozongehalt jeweils 1,9 mmol/min bei einer Gasströmungsgeschwindigkeit von 50 1/min. Durch iodometrische Titration wurde sowohl der Ozongehalt bestimmt als auch die exakte Alken-Ozon 1:1-Stöchiometrie bei der Ozonidbildung abgesichert.

Derivatisierung des Cyclopent-3-en-1-ols [12] nach der Einhornvariante [20] der SchottenBaumann Reaktion: Cyclopent-3-en-1-ylbenzoat (1d), farbloses Öl, Ausb. 62\%, Kp. 59-61 ${ }^{\circ} \mathrm{C}$ /0,003 Torr, IR (Film): $v=1720(\mathrm{C}=\mathrm{O}) \mathrm{cm}^{-1}$;

Cyclopent-3-en-1-yl-(4-nitrobenzoat) (1e), farblose Kristalle aus zuerst Ethanol, dann aus Benzin A, Ausb. $80 \%$, Fp. $93^{\circ} \mathrm{C}$, IR (KBr): $v=1720(\mathrm{C}=\mathrm{O}) \mathrm{cm}^{-1}$;

Cyclopent-3-en-1-yl-(4-chlorbenzolsulfonat) (1f), blaßgelbe Kristalle aus Ether/Benzin A, Ausb. $76 \%$, Fp. $37,5{ }^{\circ} \mathrm{C}$, IR (KBr): $v=1580(\mathrm{C}=\mathrm{C}), 1350,1180\left(\mathrm{SO}_{2}\right) \mathrm{cm}^{-1} ; \mathrm{C}_{11} \mathrm{H}_{11} \mathrm{ClO}_{3} \mathrm{~S}$ (258.7) Ber. C 51.07, H 4.25 Gef. C 50.80, H 4.26.

Malonesteralkylierung mittels 1f:

Cyclopent-3-en-1-yl-malonsäuredimethylester (1g): Zu einem Gemisch von 11.5g (0.5 mol) metallischem Natrium in $200 \mathrm{ml}$ absol. Methanol wurden $97.7 \mathrm{~g}$ (0.74 mol) Malonsäuredimethylester gegeben und unter Rühren und Rückfluß 3 h erhitzt, wobei reichlich Natrium-4chlorbenzolsulfonat ausfiel. Der überwiegende Teil des Methanols wurde abdestilliert, der Rückstand mit Wasser versetzt und die organische Phase abgetrennt. Nach viermaliger 
Extraktion der wäßrigen Phase mit je $50 \mathrm{ml}$ Ether wurden die vereinigten organischen Phasen über $\mathrm{MgSO}_{4}$ getrocknet und fraktioniert destilliert. 1g wurde als farblose Flüssigkeit erhalten, Ausb. 57.5g (59\%), Kp. 69-70 ${ }^{\circ} \mathrm{C} / 0.23$ Torr; $\mathrm{C}_{10} \mathrm{H}_{14} \mathrm{O}_{4}$ (198.2) Ber. C 60.59, H 7.12 Gef. C $60.40 \mathrm{H} 7.10$.

Cyclopent-3-en-1-yl-malonsäurediethylester (1h): $\mathbf{1 h}$ wurde analog $\mathbf{1 g}$ hergestellt, als Lösungsmittel diente hier absol. Methanol; farblose Flüssigkeit, Ausb. 51\%, Kp. 83-86 $\mathrm{C} / 0,45$ Torr, vgl. [21].

\section{Ozonisierung der Cyclopentene 1c-h}

Zur Ozonisierung der Cyclopentene 1c-h wurde folgende Versuchsanordnung benutzt: Der aus dem Ozongenerator tretende Ozon/Sauerstoffstrom wurde durch eine Reaktionsapparatur bestehend aus einer Trockensäule mit gekörntem Calciumchlorid (Länge $75 \mathrm{~cm}$, Querschnitt 3 $\mathrm{cm}$ ), einer Sicherheitswaschflasche, dem Reaktionsgefäß, einer zweiten Sicherheitswaschflasche und einer Waschflasche mit gesättigter wäßriger Natriumiodidlösung geleitet. Der Nettoozonverbrauch wurde nach jeder Ozonolyse durch Titration der ausgeschiedenen Iodmenge mittels 0,1 N Thiosulfatlösung ermittelt. Die Bestimmung des Peroxidgehaltes in der Reaktionslösung erfolgte durch Reduktion mittels essigsaurer wäßrig methanolischer Kaliumiodidlösung und Titration des ausgeschiedenen Iods wie zuvor. Alle hier eingesetzten Cyclopentene nahmen in Frigen 11 oder Methylenchlorid bei $-78{ }^{\circ} \mathrm{C}$ die äquivalente Menge Ozon auf, 1c-e bildeten jedoch keine in Substanz isolierbaren monomeren Ozonide. Im wesentlichen wurden die gleichen Beobachtungen wie bei der Ozonolyse des Norbornens [20], eines 3.5-über- brückten Cyclopentens, gemacht, die auf die Bildung polymerer Peroxide beim Versuch der Isolierung schließen ließen. Während jedoch aus 1c gemäß ${ }^{1} \mathrm{H}-\mathrm{NMR}-\mathrm{Messungen}$ 2c bei tiefer Temperatur als alleiniges Produkt erschien, waren die aus 1d,e gebildeten Ozonide 2d,e schon partiell zu den entsprechenden Aldehydcarbonsäuren isomerisiert (Aldehydprotonensignale bei $\delta=9.7-9.8 \mathrm{ppm}$, unspezifisch breite $\mathrm{OH}$-Absorption bei ca. 12.3 ppm). Eingesetzte Mengenverhältnisse: $1.8 \mathrm{~g}$ (14.3 mmol) 1c in $75 \mathrm{ml}$ Frigen 11; $1.9 \mathrm{~g}$ (10 mmol) 1d in $70 \mathrm{ml}$ Frigen 11 bzw. $3.8 \mathrm{~g}$ (20 mmol) 1 d in $150 \mathrm{ml}$ Methylenchlorid; $2.3 \mathrm{~g}$ (10 mmol) 1e in $100 \mathrm{ml}$ Frigen 11 bzw. 4.7 g (20 mmol) 1e in $150 \mathrm{ml}$ Methylenchlorid; während der Ozonolysen bildeten sich in letzteren Fällen neben der gelösten Substanz farblose Trübungen, bei denen es sich um polymere Peroxide handelte, die wegen Explosionsneigung in Substanz nicht näher untersucht wurden. 
6,7,8-Trioxa-bicyclo[3.2.1]oct-3-yl-(4-chlorbenzolsulfonat) (2f): $5.2 \mathrm{~g}(20 \mathrm{mmol}) \mathbf{1 f}$ in $70 \mathrm{ml}$ Frigen 11 (bzw. $10.4 \mathrm{~g}$ (40 mmol) in $100 \mathrm{ml}$ Methylenchlorid) wurden bei $-78{ }^{\circ} \mathrm{C}$ bis zur ersten Blaufärbung mit Ozon versetzt. Der nicht umgesetzte Ozonanteil wurde im Sauerstoffstrom nach Abschalten der Stromquelle in die Kaliumiodidlösung übergetrieben und die ausgeschiedene Iodmenge titriert. Das Lösungsmittel wurde aus dem Reaktionsgemisch abdestilliert und der Rückstand aus Ethylacetat umkristallisiert; farblose Kristalle, Ausbeute 3.3 g (54\%) (bzw. $6.2 \mathrm{~g}(50 \%)$ ), Zersetzung oberhalb $40^{\circ} \mathrm{C}$; IR $(\mathrm{KBr}): \mathrm{v}=1370,1190\left(\mathrm{SO}_{2}\right) \mathrm{cm}^{-1}$; $\mathrm{C}_{11} \mathrm{H}_{11} \mathrm{ClO}_{6} \mathrm{~S}$ (306.7, DCI-MS einer Lösung in Ethylacetat, Reaktandgas i-Butan: m/e 306 (29.5\%), 308 (5.8\%)) Ber. C 43.08, H 3.62 Gef. C 43.20 H 3.70; NMR-Daten siehe Tab. 1.

6,7,8-Trioxabicyclo[3.2.1]oct-3-yl-malonsäuredimethylester $(\mathbf{2 g}): 4.0 \mathrm{~g}$ (20 $\mathrm{mmol}) \mathbf{1 g}$ in je $70 \mathrm{ml}$ Frigen 11, Methylenchlorid oder Chloroform wurden wie zuvor umgesetzt; farblose Kristalle, Ausb. 4.9 g (quant.), Fp. $65{ }^{\circ} \mathrm{C}$; IR (KBr): v = $1740(\mathrm{C}=\mathrm{O}) \mathrm{cm}^{-1} ; \mathrm{C}_{10} \mathrm{H}_{14} \mathrm{O}_{7}(246.2$, DCI-MS wie zuvor: m/e 247 (16\%) (M+1), EI-MS: M-34 höchster Massenpeak) Ber. C 48.78, H 5.73 Gef. C 48.70, H 5.75.

6,7,8-Trioxabicyclo[3.2.1]oct-3-yl-malonsäurediethylester (2h): $4.5 \mathrm{~g}$ (20 mmol) $\mathbf{1 h}$ wurde wie zuvor $1 \mathrm{~g}$ behandelt; farblose Kristalle, Ausb. $5.5 \mathrm{~g}$ (quant.), Fp. $37^{\circ} \mathrm{C}$; IR (KBr): $v=1740$ $(\mathrm{C}=\mathrm{O}) \mathrm{cm}^{-1} ; \mathrm{C}_{12} \mathrm{H}_{18} \mathrm{O}_{7}(274.3)$ Ber. C 52.55, H 6.62 Gef. C 52.50, H 6.61.

Tabelle 1 Charakteristische NMR-Methinvergleichsdaten der Cyclopentenozonide 2d-h [22]

\begin{tabular}{|c|c|c|c|}
\hline \multirow[t]{2}{*}{2} & \multicolumn{2}{|c|}{${ }^{1} \mathrm{H}-\mathrm{NMR}^{\mathrm{a})}$} & ${ }^{13} \mathrm{C}-\mathrm{NMR}{ }^{\mathrm{a})}$ \\
\hline & CH-Ozonid & $\mathrm{CH}-\mathrm{R}^{3}$ & CH-Ozonid \\
\hline d & \multicolumn{2}{|c|}{$5.89-5.82(\mathrm{~m}, 3 \mathrm{H})$} & 99.5 \\
\hline $\mathbf{e}$ & \multicolumn{2}{|c|}{$5.95-5.30(\mathrm{~m}, 3 \mathrm{H})$} & 100.7 \\
\hline $\mathbf{f}$ & $5.80(\mathrm{~m}, 2 \mathrm{H})$ & $4.95(\mathrm{~m}, 1 \mathrm{H})$ & 98.5 \\
\hline $\mathbf{g}$ & $5.79(\mathrm{~s}, 2 \mathrm{H})^{\mathrm{b})}$ & c) & 100.0 \\
\hline h & $5.80(\mathrm{~s}, 2 \mathrm{H})^{\mathrm{b})}$ & c) & d) \\
\hline
\end{tabular}

a) Lösungen in $\mathrm{CDCl}_{3} / \mathrm{CFCl}_{3} / \mathrm{TMS}, \delta$ [ppm]; Gerät Bruker AM400, Meßfrequenz $400 \mathrm{MHz}$ $\left({ }^{1} \mathrm{H}\right)$ bzw. $100 \mathrm{MHz}\left({ }^{13} \mathrm{C}\right)$

b) Nur in $\mathbf{2 g}$, $\mathbf{h}$ wirkt der Substituent $\mathrm{R}^{3}$ so konformationsstablisierend, daß die Ozonidbrückenkopf-Wasserstoffe als scharfes Singulett erscheinen; Messungen in $\mathrm{CDCl}_{3} / \mathrm{TMS}$

c) starke Signalüberlappung

d) nicht gemessen 


\section{Literatur}

[1] R. Criegee, A. Banciu, H. Keul, Chem. Ber. 108 (1975) 1642

[2] R. Criegee, G. Blust, G. Lohaus, Liebigs Ann. Chem. 583 (1953) 1

[3] 7\% Cyclopentenozonid bei Tieftemperaturozonolyse von Cyclopenten (1a) in Pentan nach [2], dagegen 80\%, wenn 1a zuvor an hochtemperaturentwässertes Kieselgel adsorbiert worden war: I.E. Den Besten, T.H. Kinstle, J. Am. Chem. Soc. 102 (1980) 5969

[4] R. Criegee, Chimia 22 (1968) 392

[5] R.W. Murray, W. Kong, S.N. Rajadhyaksha, J. Org. Chem. 58 (1993) 315

[6] R. Criegee, G. Lohaus, Chem. Ber. 86 (1953) 1

[7] a) R. Criegee, Angew. Chem. 87 (1975) 765; Angew. Chem., Int. Ed. Engl. 14 (1975) 745

b) P.S. Bailey, in Ozonation in Organic Chemistry, Vol. I (1978), Vol. II, Chapt. XII (1982), Academic Press, New York, London

[8] a) J. Castonguay, M. Bertrand, J. Carles, S. Fliszár, Y. Rousseau, Can. J. Chem. 47 (1969) 919;

b) Y.-S. Hon, S.-W. Lin, Y.-J. Chen, Synth. Commun 23 (1993) 1543

[9] a) S.J. Rhoads, J. Chattapadhyay, E.E. Waali, J. Org. Chem. 35 (1979) 3352;

b) Ozonolyse: M. Kawaguchi, O. Hayashi, N. Sakai, M. Hamada, Y. Yamamoto, J. Oda, Agric. Biol. Chem. 50 (1986) 3107; Chem. Abstr. 108 (1988) 167736e

[10] S. Pistorius, Dissertation, S. 79, 156, Universität des Saarlandes, Saarbrücken 1992

[11] K. Schank, F. Werner, unveröffentlichte Ergebnisse aus der Diplomarbeit F. Werner, Universität des Saarlandes, Saarbrücken 1976

[12] H.M. Hess, H.C. Brown, J. Org. Chem. 32 (1967) 4138

[13] A.A. Gorman, M.A.J. Rodgers, Chem. Soc. Rev. 10 (1981) 205 auf S. 212

[14] P.S. Bailey, Chem. Ber. 87 (1954) 993

[15] R.H. Perry, Jr., J. Org. Chem. 24 (1959) 829

[16] Ozonide substituierter Norbornene: K. Griesbaum, P. Krieger-Beck, J. Beck, J. Org. Chem. 56 (1991) 4005

[17] Y. Kobayashi, I. Kumadaki, A. Ohsawa, M. Honda, Y. Iitaka, Tetrahedron Lett. 1975, 3001 
[18] Alternativvorschlag, der sich am Syntheseprinzip 2 orientiert, vgl. K. Schank, S. Moschel, Phosphorus, Sulfur, and Silicon 74 (1993) 419

[19] W. Walisch, Chem. Ber. 94 (1961) 2314

[20] H.T. Openshaw in A Laboratory Manual of Qualitative Organic Analysis, $3^{\text {rd }}$ Edit., p. 36, Cambridge at the University Press 1965

[21] C.C. Lee, E.W.C. Wong, Tetrahedron 21 (1965) 539

[22] NMR-Vergleichsdaten: $2 \mathbf{b}, \delta=5.93$ (s, 1H); 103.5 [8b] und 2c: 5.82 (d, 1H, J = 1.1 $\mathrm{Hz}) ; 104.1 \mathrm{ppm}[10]$

Korrespondenzanschrift:

Prof. Dr. K. Schank, Fachrichtung 11.2 Organische Chemie, Universität des Saarlandes, 66041 Saarbrücken, Germany 